\title{
INTERSECTIONS OF CONTRACTIBLE POLYHEDRA
}

\author{
EDWARD G. BEGLE ${ }^{1}$
}

Let the finite polyhedron $C$ be expressed as a sum of two polyhedra, $A$ and $B$. Using the Mayer-Vietoris formula, it is easy to prove the following two propositions:

(1) If $A \cap B$, the intersection of $A$ and $B$, is acyclic, ${ }^{2}$ then $C$ is acyclic if and only if both $A$ and $B$ are acyclic.

(2) If $A, B$, and $C$ are all acyclic, so is $A \cap B$.

Aronszajn and Borsuk have shown ${ }^{3}$ that proposition (1) is also true if acyclic is replaced by contractible. ${ }^{4}$ They left open the question as to whether or not this is true for proposition (2). We show by means of an example that it is not true. The example is constructed as follows:

Let $P$ be a Poincaré sphere, that is, a 3 -dimensional polyhedron with the homology groups of a 3 -sphere and with a non-vanishing fundamental group. Let $K$ be the polyhedron obtained by removing an open 3-simplex from $P$. It is easy to see that $K$ is acyclic and that its fundamental group is the same as that of $P$, so that $K$ is not contractible.

Let $C$ be the join ${ }^{5}$ of $K$ with two points, $q$ and $q^{\prime}$. Denote the join of $K$ with $q$ by $A$, and the join of $K$ with $q^{\prime}$ by $B$. Let $C=A \cup B$ the sum of $A$ and $B$. Then $A \cap B=K$. It is clear that $A$ can be continuously deformed into the point $q$, and $B$ into $q^{\prime}$, so both these polyhedra are contractible. Hence it remains to show only that $C$ is contractible. By proposition (1), $C$ is acyclic. Hurewicz has shown ${ }^{6}$ that if the fundamental group of an acyclic polyhedron vanishes,

Presented to the Society, April 18, 1942; received by the editors July 2, 1942.

1 National Research Fellow.

${ }^{2}$ A polyhedron is said to be acyclic if all its homology groups vanish for all coefficient groups.

${ }^{3}$ N. Aronszajn, and K. Borsuk, Sur la somme et le produit combinatoire des retracts absolus, Fund. Math. vol. 18 (1932) pp. 193-197.

${ }^{4} \mathrm{~A}$ set is said to be contractible if it can be deformed over itself into a point. A contractible polyhedron is acyclic.

${ }^{5}$ The join of two sets, $X$ and $Y$, is the collection of line segments joining each point of $X$ with each point of $Y$ and disjoint except for their common end points. If $X$ and $Y$ are polyhedra, so is their join.

${ }^{6} \mathrm{~W}$. Hurewicz, Beiträge zur Topologie der Deformationen, Neder. Akad. Wetensch. vol. 38 (1935) pp. 521-528, Theorem IV. 
then the polyhedron is contractible. Therefore we are reduced to showing that the fundamental group of $C$ is trivial. Let the origin for this group be chosen in $K$. Using the fact that $C$ is a polyhedron, it is easy to see that each closed path in $C$ is homotopic to a sum of closed paths, each one entirely in $A$ or in $B$. But both $A$ and $B$ are contractible, so each of these paths is homotopic to a point, which completes the proof. ${ }^{7}$

Remarks. I. ${ }^{8}$ The dimension of the polyhedron $C$ is 4 , but a similar example can be constructed for which the dimension is 3 . This follows from the fact that $K$ can be retracted by deformation into a part, $K^{\prime}$, of its 2-dimensional skeleton. Using $K^{\prime}$ in place of $K$ in the above construction, the resulting polyhedron will be of dimension 3 .

II. By the usual method of condensation of singularities, compact metric spaces $A^{\prime}$ and $B^{\prime}$ can be constructed such that $A^{\prime}, B^{\prime}$, and $A^{\prime} \cup B^{\prime}$ are all locally contractible, ${ }^{9}$ while $A^{\prime} \cap B^{\prime}$ is not. However, it is relatively easy to prove the generalization of proposition (2) to the case of locally acyclic compact metric spaces.

UNIVERSITY OF Michigan

${ }^{7}$ Cf. $\$ 52$ of H. Seifert and W. Threlfall, Lehrbuch der Topologie, Leipzig, 1934.

8 This remark is due to $\mathrm{S}$. Eilenberg.

${ }^{2}$ A space is said to be locally contractible at a point $p$ if each neighborhood $U$ of $p$ contains a neighborhood $V$ of $p$ such that $V$ can be deformed inside $U$ into a point. The space is locally contractible if it is locally contractible at each point. The definition of a locally acyclic space is entirely analogous. 